\title{
The essence of noise in nature with reference to acoustics
}

\author{
G. Rosenhouse \\ Faculty of Civil Engineering - Technion, Haifa, Israel
}

\begin{abstract}
Noise appears in all areas of science and technology, and involves everything in life. The paper shows that noise represents life itself, because it is not deterministic, and because it involves uncertainty. It can be described by mathematical formulation that illustrates various topics in an abstract way which leads to general conclusions. The paper reviews noise that exists in nature and in technology, such as molecular Brownian motion, stochastic resonance, stock market fluctuations including similarity, stochastic fractals, "random walk theories, movement ecology, and others. A list of some applications of positive noise" concludes the paper.

Keywords: noise, nature, uncertainty, random processes, noise colours, noise applications.

So far as the laws of mathematics refer to reality, they are not certain and as far as they are certain, they do not refer to reality

Albert Einstein
\end{abstract}

\section{Introduction: positive and negative kinds of noise}

Living creatures are exposed to ever increasing levels of intruding noise, due to technological advances. Negative sounds include environmental noise due to short distances between homes and main roads, industrial premises and areas, noises radiating from recreation areas or noisy neighbours and noise generated in electronic units and components of electrical circuits. Engineering solutions aim at reducing negative noise sources below threshold of annoyance or damage.

However, noise has a much wider definition than intruding stochastic or tonal sound signals. Noise is a basic entity in the essence of life. It exists everywhere 
in nature. The paper describes some aspects of noise in all areas of science and technology.

\section{Some basic definitions}

A stochastic or a chance phenomenon leads to an empirical result, related to its observation for a given set of circumstances. It generally does not yield an identical set of outputs, and thus it lacks deterministic regularity. However, it is related to a statistical regularity of various outputs.

Thus, the relative frequency of observation of various possible outputs in a set of arbitrary observations (or a random choice) to events that are independent of each other is a number between zero and one - see figure 1 .

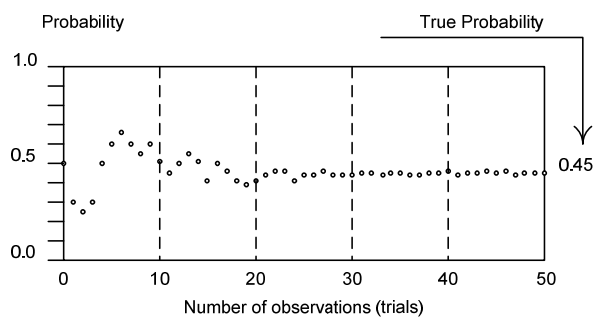

Figure 1: A calculated probability as a function of the number of trials.

\section{Gaussian noise}

Gaussian noise is a most widespread bell shaped probability distribution in nature. When it is filtered by a linear filter it keeps Gaussian expected amplitude distribution. The sum of random Gaussian variables still keeps a Gaussian distribution. The discrete Gaussian distribution is:

$$
\mathrm{p}\left(\mathrm{x}_{\mathrm{i}}\right)=\frac{1}{\sigma \sqrt{2 \pi}} \exp \left[\frac{-\left(\mathrm{x}_{\mathrm{i}}-\mathrm{m}\right)^{2}}{2 \sigma^{2}}\right], \mathrm{m}=\frac{\sum_{\mathrm{i}=1}^{\mathrm{N}} \mathrm{x}_{\mathrm{i}}}{\mathrm{N}}, \sigma=\sqrt{\frac{\sum_{\mathrm{i}=1}^{\mathrm{N}}\left(\mathrm{x}_{\mathrm{i}}-\mathrm{m}\right)^{2}}{\mathrm{~N}}}
$$

$\mathrm{x}_{\mathrm{i}}$ is a certain chosen value $\mathrm{i}$ of $\mathrm{x}, \mathrm{m}$ is the mean or nominal value and $\sigma$ is the standard deviation. It means dependence on two parameters, $m$ and $\sigma$ only and being symmetrical.

\section{Acoustics and colours}

In the $17^{\text {th }}$ Century Newton investigated the combined sensation of colour and sound. He assumed that musical and colour tones had common frequencies. Synaesthesia is a neurological phenomenon in which stimulation of a sensory/cognitive pathway causes automatic forced experience in another 
sensory/cognitive pathway. A broadband synaesthesia involves a variety of environmental sounds where each component of colour can change. Brightness in this context is the amount of white in the colour - when brightness is eliminated from red colour it becomes brown and finally black.

\section{White noise}

"White noise" (WN) is a process characterized by lack of correlation at two different times, but its power spectral density is flat over the whole range of frequency bands. Acoustic WN contains equal quantities of intensity at all heard frequencies. Each amplitude has the same chance to be at a certain point as any other amplitude. White light includes equally all the visible colours, and as a result it is a broad band noise. Daylight, having all the visible colours is noise. It means - we live in noise (electromagnetic and acoustic). There is frequently interest in Gaussian distribution of the acoustic power and major difference between distributions is the thickness of their "tails". A thicker tail yields more extreme events [1]. A form of a WN is Johnson's noise [2,3] which results from a thermal excitation of electrical components, such as a resistor. Noise is caused by the motion of atoms charge which makes the resistor. The thermal excitation is caused by heat and when the resistor gets hotter it becomes noisier. Many tests of electrical equipment and components use various colours: white, pink or brown, and researchers have been applying various standard noise colours for different acoustic measurements. See figs 2-3.

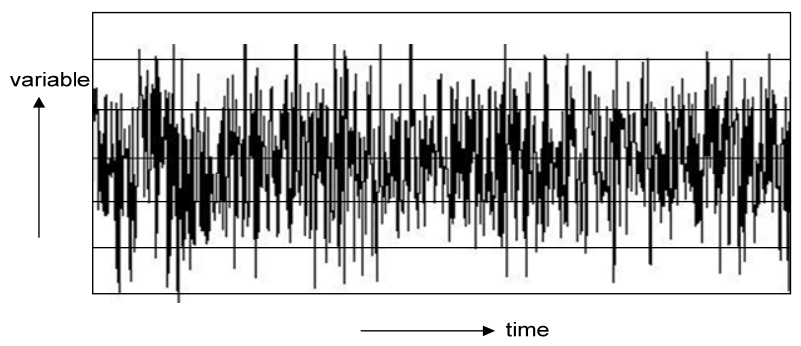

Figure 2: A typical plot of a white noise.

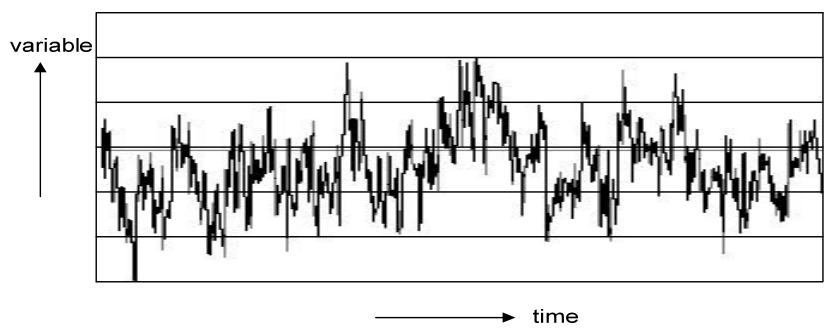

Figure 3: A typical plot of a pink noise. 
Johnson [3] discovered noise scales, where the random process $S(f)$ is given by the following general dependence on frequency.

$$
S(f)=\frac{C}{f^{\alpha}} ; \mathrm{C} \text { is a constant } \mathrm{f} \text { is the frequency in Hertz. }
$$

Many phenomena in physics, astrophysics, geophysics, biology, technology, speech and music, economics and psychology are at the range $0.5 \leq \alpha \leq 1.5$. The different colours (white, pink, brown and others) have different coefficients, $\alpha$.

\section{Gaussian white noise (GWN)}

In the case of Gaussian white noise the mean or the expected value is zero: $\mathrm{m}=\mathrm{E}(\mathrm{x})=\int_{-\infty}^{\infty}[\mathrm{xp}(\mathrm{x})] \mathrm{dx}=0$, and the mean standard deviation, $\sigma$ of the pseudo random sequence becomes: $\sigma=\sqrt{\mathrm{E}(\mathrm{x}-\mathrm{m})^{2}}=\sqrt{\mathrm{E}(\mathrm{x})^{2}}$, for $\mathrm{m}=0 . \mathrm{x}$ is a random variable defined on a probability space. $\mathrm{p}(\mathrm{x})$ is the probability density function (pdf). If $\mathrm{x}$ is a discrete random variable, then a probability mass function (pmf), $\mathrm{f}(\mathrm{x})$ is used and: $\mathrm{E}(\mathrm{x})=\sum_{\mathrm{i}}[\mathrm{x} \mathrm{p}(\mathrm{x})] \mathrm{dx}=0 ; \quad \sigma=\sqrt{E(x-m)^{2}}=\sqrt{E(x)^{2}}$, for $\mathrm{m}=0$.

GWN provides simulation of real situations in our world. An additive white Gaussian noise (AWGN) channel is regularly used in communication. The input signal $\mathrm{s}(\mathrm{t})$ is contaminated by noise, $\mathrm{n}(\mathrm{t})$, that can be AWGN, during its transmission from the source location to the receiver, and it becomes distorted in amplitude and phase, including a varying time delay. The result is the output:

$$
y(t)=s(t)+n(t), \text { see figure } 4
$$

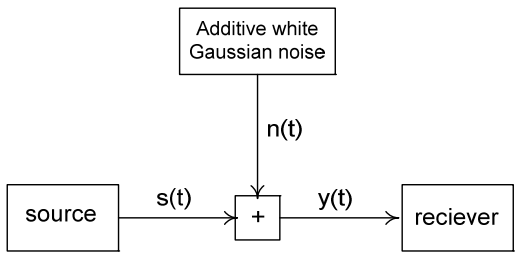

Figure 4: A signal along a communication line, combined + an undesired WN.

\section{Pink noise}

Pink noise depends on the ratio $1 / \mathrm{f}$. Its bandwidth ranges up to $20 \mathrm{kHz}-$ See figures 5:

$$
\mathrm{S}(\mathrm{f})=\frac{\mathrm{C}}{\mathrm{f}^{\alpha}} ; \alpha=1 \therefore \mathrm{S}(\mathrm{f})=\frac{\mathrm{C}}{\mathrm{f}} ; \mathrm{C} \text { is a constant }
$$



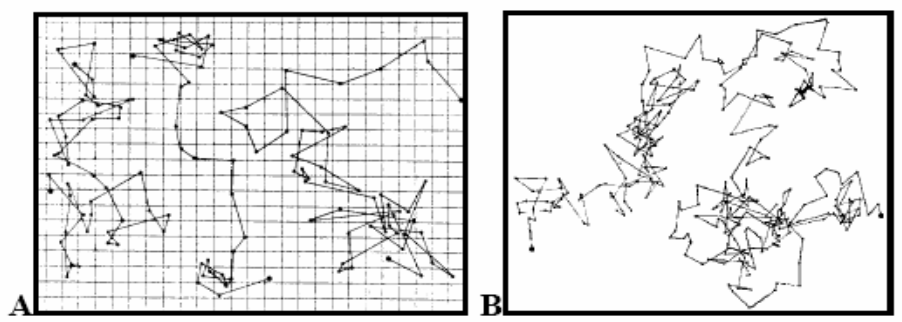

Figure 5: $\quad$ Brownian motion as measured by Perrin.

As a result of this dependence, pink noise has a uniform distribution along the logarithmic frequency scale, which means a flat spectral density as a function of the percentage of the band width - or an equal power for each octave band. It means that pink noise is a frequency spectrum whose intensity decays at approximately the rate of $3 \mathrm{~dB}$ per octave (or $10 \mathrm{~dB}$ per each decade). Examples in nature include brain EEG, brain MEG, human heart sound, human time estimation, squid giant axon, vacuum tube and semiconductor noise, music and natural sounds, and applications in sound and audio systems and tests. Pink noise is very popular in testing building and environmental acoustics. The flicker noise [4], which is $1 / \mathrm{f}$ noise, is very close to pink noise. It is used in solid state physics and it describes noise radiated from defects along the wave-guide channel, as a result of base currents in conductors.

\section{Brown or Brownian noise (Brownian motion - BM)}

The term Brownian noise is coined after Robert Brown (1773-1858) who discovered Brownian motion, which is the stochastic motion of particles (pollen) that float over a fluid - published in 1828. During BM each new point is moved stochastically a little bit from the previous state due to a "random walk" (RW) a term originated by Karl Pearson [5]. Brown noise is the integral of white noise. Its definition is:

$$
\mathrm{S}(\mathrm{f})=\frac{\mathrm{C}}{\mathrm{f}^{\alpha}} ; \alpha=2 \therefore \mathrm{S}(\mathrm{f})=\frac{\mathrm{C}}{\mathrm{f}^{2}} \quad \mathrm{C} \text { is a constant }
$$

Brown noise expresses a drop of $6 \mathrm{~dB}$ per octave band - more energy at the lower frequencies - it means a roar of low frequencies. BM theory is used in areas such as molecular biology, wireless nets and changes in the stock market.

\section{From an assumption of a world of atoms and molecules to a theoretical proof of their existence through BM}

For Einstein (1877-1955) BM was the opportunity to prove the existence of atoms and molecules. Einstein showed that van't Hoff (1852-1911) law concerning osmotic pressure (1884) was a direct consequence of the kinetic molecular theory of heat, satisfied also in dilute particle systems. He then went on to develop the expression for the average square root of displacement of 
particles floating over a fluid. Figure 5 shows an example of Perrin's measurement of Brownian motion.

Einstein's theory and Perrin's observations have shown that the average distance of a floating particle increases in proportion to the square root of time were:

$$
\mathrm{x} \propto \sqrt{\mathrm{t}} .
$$

A simple form of Brownian motion is the Wiener equation, in which the instantaneous fluid particle velocity $\overrightarrow{\mathrm{V}}$ that fluctuates randomly is:

$$
\vec{v}(t)=\frac{d \vec{x}(t)}{d t}=\vec{g}(t)
$$

$\overrightarrow{\mathrm{X}}$ is the position of the particle and $\overrightarrow{\mathrm{g}}(\mathrm{t})$ can be WN. Wiener's equation is not suitable for short time scales due to instantaneous velocity change. For short time scales Langevin equation which considers particle acceleration is needed.

\section{The economic aspect of the Brownian movement}

Thiele (1838-1910) was the first to model mathematically BM with independent and normally distributed increments and variances proportional to time, while studying time series [6]. IN BM, the position of a particle $\mathbf{x}(\mathrm{t})$ at the time $\mathrm{t}$ is a result of a random process. The change of location in each step leads to a new location as follows:

$$
\mathbf{x}(\mathrm{t}+\Delta \mathrm{t})=\mathbf{x}(\mathrm{t})+\mathbf{v} \Delta \mathrm{t}^{0.5} \mathrm{~N}(0,1)
$$

$\mathbf{v}$ is the average speed of a particle and $\mathrm{N}(0,1)$ is a normal randomly distributed variable. The increment $\mathbf{x}\left(\mathrm{t}_{2}\right)-\mathbf{x}\left(\mathrm{t}_{1}\right)$ has a Gaussian distribution, with the average $\mathrm{E}$ and the variance $=\sigma^{2}(\sigma$ - standard deviation $)$ properties as follows:

$$
\mathrm{E}\left[\mathrm{x}\left(\mathrm{t}_{2}\right)-\mathrm{x}\left(\mathrm{t}_{1}\right)\right]=0 ; \operatorname{Var}\left[\mathrm{x}\left(\mathrm{t}_{2}\right)-\mathrm{x}\left(\mathrm{t}_{1}\right)\right] \propto\left|\mathrm{t}_{2}-\mathrm{t}_{1}\right| .
$$

The position of a particle $\mathbf{x}(\mathrm{t})$ is continuous, but not differentiable. The increments: $\mathrm{x}\left(\mathrm{t}_{0}+\mathrm{t}\right)-\mathrm{x}\left(\mathrm{t}_{0}\right)$ and $\frac{\left[\mathrm{x}\left(\mathrm{t}_{0}+\mathrm{rt}\right)-\mathrm{x}\left(\mathrm{t}_{0}\right)\right]}{\mathrm{r}^{0.5}}$ are statistically self similar. Also: If $\mathrm{t}_{0}=0, \mathrm{x}\left(\mathrm{t}_{0}\right)=0$, then, $\mathrm{x}(\mathrm{t})$ and $\frac{\mathrm{x}(\mathrm{rt})}{\mathrm{r}^{0.5}}$ are statistically equivalent.

The last assumptions allow for development of the algorithm that generates 1D Brownian motion.

The fractional Brownian motion ( $\mathrm{fBm})$ is a continuous Gaussian process $B_{H}(t)$, on $[0, T]$. It starts at zero and has zero expectation for all $t$ 's in this domain. In fact it is an extension of the Brownian motion $(\mathrm{Bm})$, which carries the form:

$$
\operatorname{var}\left[x\left(t_{2}\right)-x\left(t_{1}\right)\right] \propto\left|t_{2}-t_{1}\right|^{2 H} \text { and }
$$


$\mathrm{x}(\mathrm{t})$ and $\mathrm{r}^{\mathrm{H}} \mathrm{x}(\mathrm{rt})$ are statistically self similar with respect to the Hurst parameter (or index), which is a real number associated with $\mathrm{fBm}$. Thus, the $\mathrm{fBm}$ is properly rescaled by dividing the amplitudes by $\mathrm{r}^{\mathrm{H}}$.

$0 \leq \mathrm{H} \leq 1$ determines the roughness of the curve.

$\mathrm{H}$, which associates with $\mathrm{fBm}$ is a real number named after Hurst, introduced by Mandelbrot and van Ness. A graph of statistical processes of various Hurst parameters is given in figure 6 .

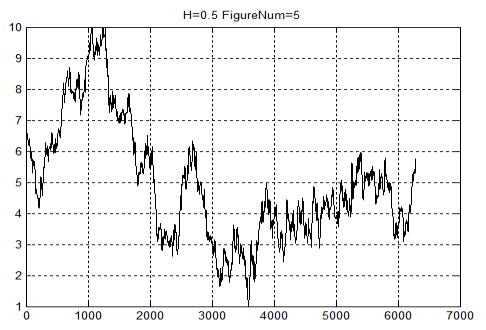

Figure 6: An example of Hurst parameter $=0.5-$ performed by Daphne Sobolev.

If $\mathrm{H}=0.5$ then $\mathrm{BM}$ occurs with independent and uncorrelated increments. $\mathrm{H}>0.5$ yields smooth curves and positive correlated increments. $\mathrm{H}<0.5$ results in erratic rough curves and increments with negative correlation.

The increment $\Delta \mathrm{B}_{H}(\mathrm{t})=\mathrm{B}_{\mathrm{H}}(\mathrm{t}-1)-\mathrm{B}_{\mathrm{H}}(\mathrm{t})$ is the fractional Brownian noise.

The $\mathrm{fBm}$ satisfies the following covariance function:

$$
E\left[B_{H}(t) B_{H}\left(t_{1}\right)\right]=\frac{1}{2}\left[|t|^{2 \mathrm{H}}+\left|t_{1}\right|^{2 H}-\left|t-t_{1}\right|^{2 H}\right]
$$

The fBm of the Weyl type (in Mandelbrot and van Ness), is defined as:

$$
\mathrm{B}_{\mathrm{H}}(\mathrm{t})-\mathrm{B}_{\mathrm{H}}(0)=\frac{1}{\Gamma\left(\mathrm{H}+\frac{1}{2}\right)}\left\{\begin{array}{l}
\int_{-\infty}^{0}\left[\left(\mathrm{t}-\mathrm{t}_{1}\right)^{\mathrm{H}-0.5}-\left(-\mathrm{t}_{1}\right)^{\mathrm{H}-0.5}\right] \mathrm{dB}\left(\mathrm{t}_{1}\right)+ \\
\int_{0}^{\mathrm{t}}\left[\left(\mathrm{t}-\mathrm{t}_{1}\right)^{\mathrm{H}-0.5} \mathrm{~dB}\left(\mathrm{t}_{1}\right)\right]
\end{array}\right\},
$$

for $\mathrm{t}>0$ and for $\mathrm{t}<0$.

Both $\mathrm{fBm}$ of the Riemanian - Liouville type and the one of Weyl type are self similar, having the property:

$$
\mathrm{B}_{\mathrm{H}}(\mathrm{at}) \equiv \mathrm{a}^{\mathrm{H}} \mathrm{B}_{\mathrm{H}}(\mathrm{t}) ; \mathrm{a}>0 ;
$$

The symbol $\equiv$ in eqn. (14) is equality in the stochastic sense. Hence, Weyl integral is a fractional integral of white noise, used to define the BM process. 
Bachelier found the stochastic process of BM. In his thesis "Théorie de la Spéculation" he presented an approach that was the trigger to the science of finance mathematics. He modelled BM to estimate the stock market changes, leading to the Black and Scholes [7] option pricing theory. The BM RW is based on the mutual dependence of two random variables, e.g. Gaussian distribution:

$$
\mathrm{p}\left(\mathrm{x}_{\mathrm{i}}, \mathrm{m}, \sigma\right)=\frac{1}{\sigma \sqrt{2 \pi}} \exp \left[\frac{-\left(\mathrm{x}_{\mathrm{i}}-\mathrm{m}\right)^{2}}{2 \sigma^{2}}\right]
$$

It stimulated the forecast that stock market behaved as a wave principle suggested by Elliott (1871-1948). An example of price changes is in figure 7 . Price changes show stochastic fractals behaviour and self similarity. See fig. 10.

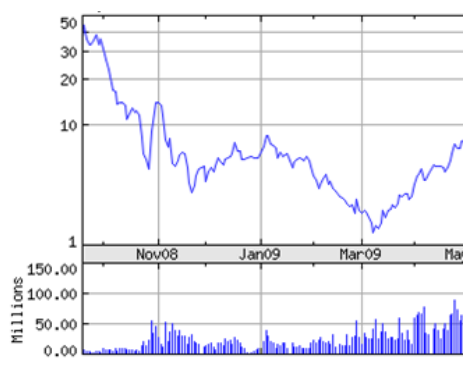

Figure 7: An example of price changes of a stock.

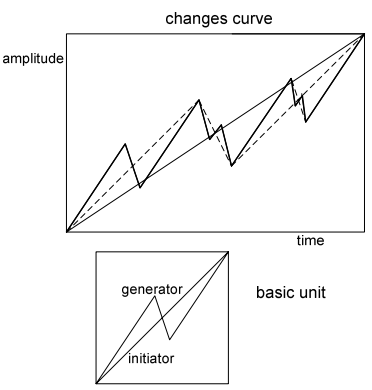

Figure 8: $\quad$ Self similar and self affine systems.

\section{Intentional random movement in nature}

The plague that caused the death of many sea lions due to lack of regular navigation abilities shows the importance of a good navigation system of living creatures not only concerning prey - predator relations but as a survival issue. Animal movement has therefore become a major experimental and theoretical research topic in ecology. Theoretical tools backed by experimental evidence address individual animal motions as reacting to their environment. Such motion resembles the mathematical patterns that follow "random walk" (RW) [8]. This 
phenomenon appears at a wide range of scales. The theoretical tools used include fractal analysis, self similarity [9], first passage time, Lévy flights, Hidden Markov Models, correlated random walks, etc. Again, movement ecology shows how much life is linked to noise.

\section{Applications of noise}

\subsection{The use of noise to improve intelligibility}

Paracusis, a phenomenon known mainly in people with inner ear deafness, is that the speech hearing improves when background noise is added. This was first observed by Willis (1621-1675). This phenomenon indicates preservation of the hearing function, or the ability to hear better in noise (see Stewart [10]).

\subsection{The use of white noise components in sirens}

Some kinds of sirens use both deterministic and stochastic signals. An aspect of WN use in siren acoustic signals is to increase the security of emergency vehicles against collisions, or improving the audibility of such sirens. First, the signal has to be loud enough and include a wide frequency spectrum in the hearing sensitive domain $(1-4 \mathrm{kHz})$ to overcome masking noise. The efficacy of three common kinds of sirens was examined, where the last one included WN component in the signal [11]. The kinds of sirens were "wail", "yelp" and "Localiser". See figure 9

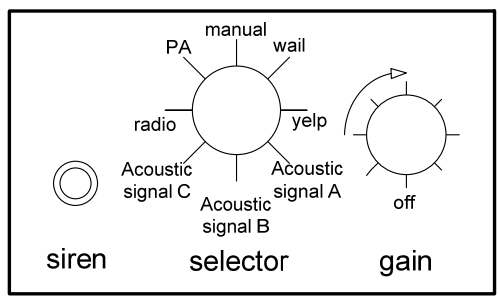

Figure 9: A scheme of an electronic siren board.

It was found that the Localiser siren had better effective range and better penetration as a result of added WN effect, since the Localiser combines yelp and white noise.

\subsection{Masking information and negative noise by noise}

Adding natural or artificial noise can mask an intruding sound or confidential information. This improves the convenience of workers and visitors in some area, say, an open space office. Commercial maskers have been developed to generate masking sounds either as loudspeakers array systems or CD recordings. Many of them are based on white or pink noise signals to mask. 
Outdoors, the effect of transportation noise will be covered by pleasant bells or waterfall sounds or special soundscape music.

\subsection{Masking tinnitus by noise}

There are many kinds of tinnitus (Rosenhouse [12], pp.1, 27, 386-395). Some of them can be relieved by masking sounds and noise. Usually, the masking noise can be based on sounds from nature, rural areas and familiar sounds. Yet WN and pink noise (due to its lower frequencies) can be used.

\subsection{The stochastic resonance}

Stochastic resonance (SR) is a non-linear dynamics process, where information flow in a multistate system is strengthened by presence of an optimal random noise. SR requires also that the signal to noise ratio (SNR) will increase. Stochastic resonance was introduced by Benzi et al. in 1981. As SR occurs when ambient noise intensity increase in a non-linear system enhances a weak periodic signal, signal reception can be improved. This effect has many applications and one example is that by activating external noise signal on crayfish mechanoreceptor cells, the individual neuron could provide physiological layer for SR in the sensory system (Douglas and Wilkens [13]).

\subsection{Active noise control}

The paper adopts the role of noise in active sound control as defined in Rosenhouse [12] (p.376): "The suggested generalized definition of ANVC (Active Sound and Vibration Control) ....... says: Active Sound (or noise, as a sub-group) and Vibration Control (ASVC) includes any creation of secondary acoustic fields in order to change intentionally the primary acoustic field in favour of acoustic or other needs. With such a broad definition, which is far from the "cancellation of noise" as defined by Lueg (1933), it can be said that "the sky is the limit.",.

In acoustics black noise is sort of a WN. It is an anti-phased WN, which as such cancels the primary $\mathrm{WN}$ by a negative overlapping of the original signal shape. It is an "anti-sound" which has a specific application of cancelling undesired noise. This is active noise control that is usually performed by electrical means.

\subsection{Environmental noise screening by leaves rustle noise}

A forest can screen the low floors of buildings from a noise source, such as transportation on a main road, by the amount of about $10 \mathrm{~dB} / 100 \mathrm{~m}$ depth depending on trees density and sizes (for a model, see [12] pp.309-329). In addition to screening, leaves rustling noise can also supply natural masking to man. This rustle noise is roughly a sort of "white noise" 


\section{Summary}

Noise is the essence of life because it is random and uncertain but not deterministic. Based on noise, the importance of atoms and molecules terminology was realized, and not the less is the basic importance of noise in our lives. Noise should be treated mathematically as any signal with certain properties that lead either to positive or negative effects on our lives, but above all it is tied to the basic definition of life itself. Noise appears as disturbance from some scientific, technological and social points of view, but it can be looked at differently - depending on the context. Some people say that the essence of life is tranquility but to say that noise is the essence of life is much stronger.

\section{References}

[1] G. Rosenhouse, "Smaller samples of the same properties are less vulnerable than larger ones", as a general rule that emerges from the "tail statistics", Int. J. of Design and Nature and Ecodynamics, WIT Press, 3, 1, 2008, 1-11.

[2] Johnson, J. B., Thermal agitation of electricity in conductors, Phys. Rev. 32, pp.97-109, 1928.

[3] Johnson, J.B., The Schottky effect in low frequency circuits, Phys. Rev. 26, pp.71-85, 1925.

[4] Barnes, J. A, and Allan, D.W., A statistical model for flicker noise, Proc. IEEE, 54(2), pp.176-178, 1966.

[5] Pearson, K., The problem of random walk, Nature, 72(1865), pp.294, 342, 27.7.1905.

[6] Thiele, T.N., Theory of observation, Dayton, London, 1903, Reprinted in Annals of Mathematical Statistics, 2, pp.165-308, 1931.

[7] Black, F. and Scholes, M., The pricing of options and corporate liabilities, J. Political Economy, 81, pp.637-659, 1973.

[8] Johnson, D.S., London, J.M., Lea, M. -A. and Durban, J., Continuous time correlated random walk model for animal telemetry data, Ecology, 89, pp.1208-1215, 2008.

[9] Turchin, P., Fractal analysis of animal movement, a critique, Ecology, 77(7), pp.2086-2090, 1996.

[10] Stewart, G.W., Introductory Acoustics, D. Van Nostrand, NY,pp.132-133, 1946.

[11] Withington, D.J., Paterson, S.E., Safer Sirens, FireEngineers Journal, 48, pp.6-10, 1998.

[12] Rosenhouse, G., Active Noise Control - fundamentals for acoustic design, Vol. I. WIT Press, Southampton and Boston, 2001.

[13] Douglas, J.K. and Wilkens, L.A. Directional selectivities of near-field filiform mechanoreceptors on the crayfish tailfan (Crustacean: Decapoda), Comp. Physiol. A., 183, pp.23-34, 1998. 